\title{
Memoria de rostros y reconocimiento emocional: Particularidades en adultos mayores institucionalizados y que conviven con sus familiares
}

\author{
Mémoire de visages et reconnaissance des émotions : les particularités des adultes aînés en résidence et d'un groupe contrôle qui \\ habite avec sa famille
}

Memória para rostos e reconhecimento emocional: Particularidades em adultos idosos institucionalizados e que convivem com seus familiares

Memory of faces and emotional recognition: Particularities in institutionalized older adults and a group who lives with their families

\section{Yunier Broche Pérez \& Luis Felipe Herrera Jiménez ${ }^{1}$}

\author{
${ }^{I}$ Universidad Central "Marta Abreu” de Las Villas, Cuba.
}

Agradecimientos: Agradecemos a Rosalía Cárdenas Rodríguez quien, pese a su juventud como investigadora, sirvió de apoyo constante durante la realización de este proyecto investigativo.

\section{Resumen}

El presente estudio estuvo dirigido a describir la memoria de rostros y el reconocimiento emocional en adultos mayores institucionalizados y no institucionalizados, que no portan demencia, residentes en la provincia de Villa Clara. Para el logro del objetivo trazado se realizó un estudio descriptivo sustentado en el paradigma cuantitativo. Fueron utilizadas como herramientas para la recogida de la información el Cambridge Memory Test for Faces y se diseñó una tarea de reconocimiento emocional. Los principales resultados muestran diferencias significativas entre ambos grupos respecto a las variables estudiadas. En relación a la memoria de rostros, los adultos mayores institucionalizados muestran un mayor deterioro en relación a los que no están internados con una significación muy alta. Sobre este aspecto, los adultos internos muestran mayores dificultades en la memoria de rostros simple, múltiple y con ruido visual, respecto a los que residen con sus familias. También se encontraron diferencias significativas, entre los grupos respecto al reconocimiento de emociones, viéndose más afectados los adultos mayores internados. Las áreas que mostraron afectación fueron el reconocimiento de emociones negativas y la decodificación de la edad. El reconocimiento del género permanece conservado en ambos grupos, sin mostrar diferencias en la exploración realizada.

Palabras claves: Adulto mayor; institucionalización; memoria de rostros; reconocimiento emocional.

\section{Résumé}

Cette étude décrit la mémoire de visages et la reconnaissance émotionnelle chez des adultes aînés, sans antécédents de démence, en résidence gériatrique et chez des aînés en cohabitation avec leur famille de la province Villa Clara, Cuba. Le « Cambridge Memory Test for Faces » (CFMT) et un test de reconnaissance des émotions ont été utilisés. Les résultats montrent des différences significatives entre les groupes par rapport aux variables étudiées. En comparaison avec les aînés qui habitent avec leur famille, la mémoire de visages des participants en résidence suggère une atteinte. De plus, les trois niveaux mesurés par le CFMT ont montré des atteintes possibles chez les aînés en résidence. En outre, des différences significatives au niveau de la reconnaissance des émotions ont été trouvées entre les groupes. Les sphères atteintes touchent la reconnaissance des émotions négatives et le décodage de l'âge. La reconnaissance du genre demeure intacte dans les deux groupes.

Mots clés: Aînés; institutionnalisation; mémoire; émotion; reconnaissance de visages.

Artículo recibido: 02/11/2011; Artículo revisado: 15/12/2011; Artículo aceptado: 22/12/2011.

Yunier Broche Pérez, Universidad Central "Marta Abreu" de Las Villas. Luis Felipe Herrera Jiménez, Universidad Central "Marta Abreu" de Las Villas.

Correspondencia relacionada con este artículo debe ser enviada a: Yunier Broche Pérez. Universidad Central "Marta Abreu" de Las Villas, Carretera de Camajuaní Km51ำ Santa Clara, Villa Clara, Cuba. CP: 54830

E-mail: yunierbp@uclv.edu.cu

DOI: $10.5579 / \mathrm{rnl} .2011 .0078$ 


\section{Resumo}

O presente estudo descreve a memória de faces e reconhecimento emocional em idosos institucionalizados e não institucionalizados sem história de demência, residentes na província de Villa Clara, Cuba. Foi utilizado o Cambridge Memory Test for Faces e uma tarefa de reconhecimento emocional foi delineada. Os resultados principais demonstraram diferenças significativas entre grupos em relação às variáveis estudadas. A memória de faces dos idosos internos demonstrou mais rápida deterioração quando comparados aos idosos que viviam com as suas famílias. Os adultos internos mostraram dificuldades maiores na memória de rostos simples, múltipla e com ruído visual, quando comparados aos que residem com as sãs famílias. Também se encontraram diferenças significativas, entre os grupos a respeito do reconhecimento de emoções, sendo mais afetados os adultos institucionalizados. As áreas que mostraram prejuízo foram o reconhecimento de emoções negativas e a decodificação da idade. O reconhecimento de gênero permaneceu preservado em ambos os grupos, sem diferenças entre grupos na investigação realizada.

Palavras-chave: Idosos; institucionalização, memória de faces; reconhecimento emocional.

\section{Abstract}

The present study describes the memory of faces and emotional recognition in elderly institutionalized and not institutionalized without history of dementia, residents in the Villa Clara province, Cuba. We used the Cambridge Memory Test for Faces and designed an emotion recognition task. The main results show significant differences between groups regarding the variables studied. The memory of faces of older intern shows further deterioration compared to the elderly living with their families. The three levels measured CFMT shows further deterioration in institutionalized adults. We also found significant differences between groups in the recognition of emotions. The areas that were affected showed the recognition of negative emotions and the decoding of age. The recognition of gender remains preserved in both groups.

Keywords: Old adults; institutionalization; faces memory; emotional recognition.

Los textos de Psicología Cognitiva y Neuropsicología abarcan profundamente el tema de la memoria como proceso cognitivo. Sin embargo los capítulos destinados a la memoria de rostros como un tipo específico de memoria son muy reducidos. Esto ha provocado que el conocimiento que se tiene hoy sobre la memoria de rostros sea relativamente poco aunque debe reconocerse que en la última década se han concluido estudios interesantes, pero todavía insuficientes. Es nuestra consideración que esto ha ocurrido, en lo fundamental, por la incorrecta noción que existió durante mucho tiempo que concebía que la memoria de caras operaba bajo los mismos mecanismos y poseía el mismo substrato que el resto de las memorias.

La memoria de rostros es una operación compleja en la que intervienen varias regiones cerebrales: la corteza visual occipital para el acceso e interpretación de la imagen, regiones frontotemporales para la organización e identificación de rasgos, el hipocampo para acceder a los recuerdos relativos a las personas que vemos y los polos temporales para acceder al nombre (Ballesteros, 1999).

Dada la importancia que tiene el recuerdo de las caras desde el principio de la vida social del hombre, la evolución ha asegurado que estas funciones estén bien consolidadas. El recuerdo e interpretación del rostro de las personas es uno de los elementos más importantes de nuestra vida de relación. Durante la vida memorizamos una gran cantidad de caras y de detalles de caras y, generalmente, nos parece sencillo el reconocerlas (Lopera, 2000).

En la memoria de las caras intervienen también aspectos de tipo emotivo: se recordarán más unas caras que otras. También intervienen factores raciales: se reconocen y recuerdan mejor las caras de los individuos de la propia raza: una persona mayor de raza caucásica reconoce mejor caras de este tipo, peor caras del norte de Europa y mucho peor caras de sujetos de raza negra o asiáticos (Bad-Jaim, Saidel, \& Yovel, 2009).

La memoria de caras se ha estudiado comúnmente con tareas de reconocimiento (Manzanero, 2010). Sin embargo, estudiar la memoria de rostros conlleva inevitablemente al estudio de las características de las caras que permiten que un rostro sea recordado. En este caso los estudios centrados en la investigación sobre reconocimiento facial de emociones son sumamente interesantes. Las particularidades del reconocimiento facial de las emociones aportan información distintiva que ayuda a comprender la compleja dinámica que se establece entre la memoria para caras y la decodificación que trasmite el rostro.

Para cualquier persona es muy obvio observar un elemento, sea un vaso, un auto, una mascota, un amigo, un familiar, e identificarlo como tal. Sin embargo el sistema funcional que subyace al reconocimiento de cualquier objeto a través de la estimulación de los órganos de de la vista, es sumamente complejo, especialmente si se trata de un rostro. La particularidad del rostro radica en su función social, puesto que a través de él es que se recibe información sobre las otras personas, aun sin estar en contacto directo con las mismas. La expresión en la cara de los demás trasmite información sobre su estado emocional, lo que nos permite tomar decisiones o regular nuestro comportamiento en el marco de las relaciones sociales. Al mismo tiempo, nos ayuda a mantener lazos interpersonales profundos puesto que somos capaces de diferenciar a nuestros familiares y amigos del resto de las personas.

Un modelo que procura explicar este mecanismo fue propuesto por Bruce y Young (1986). Según estos autores el procesamiento de rostros conocidos atraviesa por una serie de pasos, desde que el rostro es percibido hasta que se evoca el nombre de la persona reconocida. Estos procesos, son la construcción del percepto facial, reconocimiento facial, activación de la memoria semántica relativa a las personas, el acceso lexical y por último la producción articulatoria (Ver Fig. 1).

Partiendo de este modelo y teniendo en cuenta otros hallazgos Haxby, et al., (2000) propuso un sistema de distribución cortical de los subproceso involucrados en la percepción de rostros conocidos. El autor propone una estructura basada en la distinción entre los aspectos invariantes del rostro (que permite determinar la identidad) y la representación de los aspectos variables del rostro (que son aquellos que permiten el proceso socializador). El mismo 


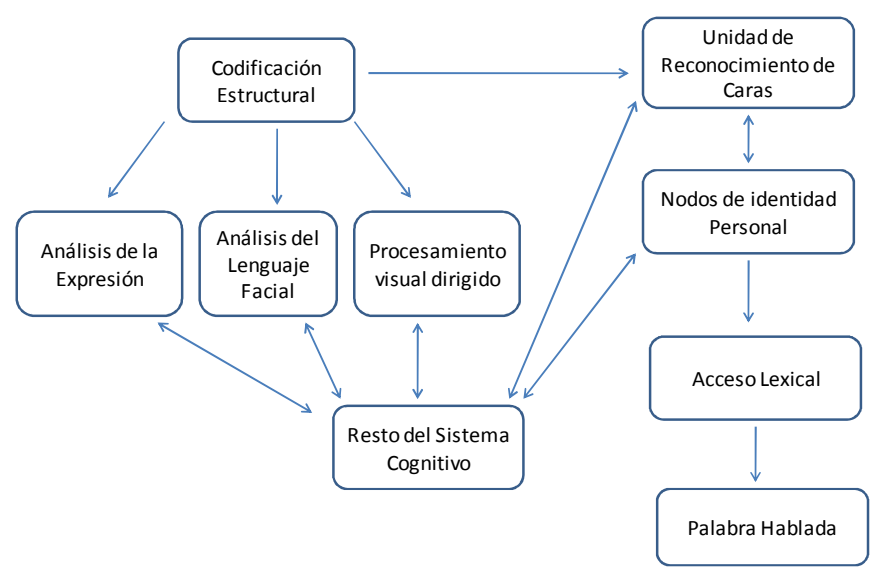

Figura 1. Modelo Funcional para el Procesamiento de Rostros de Bruce y Young

presenta una estructura jerárquica que distingue un sistema doble para el análisis visual de los rostros y otro para el proceso que permite dotar de significado la información que de las caras se obtiene.

Este sistema doble consiste en tres áreas bilaterales con una configuración anatómica que sugiere, jerárquicamente, una organización donde la región occipital inferior provee el estímulo a la zona lateral fusiforme y a las regiones del surco temporal. Adicionalmente este sistema neural tiene extensiones hacia el sistema de percepción facial. El sistema neural para la atención espacial y la percepción, con las regiones cerebrales ubicadas en el surco intraparietal, más probamente, en las áreas visual frontal, procesa la información proveniente de los rostros, como la mirada, la posición de la cabeza, para dirigir la atención.

El sistema neural para la comprensión verbal auditiva en el giro temporal superior participa en el procesamiento de la lectura de los labios, presumiblemente extrayendo la información fonética. Los sistemas neurales para representar conocimiento semántico biográfico en el lóbulo temporal anterior participan en la recuperación del nombre y otra información relacionada con el rostro. Los sistemas para el procesamiento de la emoción, identificados en la amígdala y la ínsula, procesan la información con contenido emocional. A continuación se modela la propuesta (Fig. 3).

La memoria de rostros, como proceso, no es lineal, sino dependiente de múltiples estructuras anatómicas y mecanismos funcionales, que en su accionar concertado, alcanzan el objetivo final del reconocimiento. De esta forma, los dos esquemas proveen una descripción de subsistemas funcionales que subyace a varios aspectos de la percepción facial.

La importancia fundamental del estudio tanto de la memoria de rostros como del procesamiento de caras radica en el hecho de que ambos procesos abarcan armoniosamente la relación de lo cognitivo y lo afectivo. Nuestras relaciones objetales y sociales están determinadas básicamente sobre procesos de reconocimiento, ya sean sonoros, gustativos, olfativos, táctiles o visuales. En este sentido resulta extraordinario como ambas categorías cumplen funciones tan importantes e imprescindibles, no solo en el establecimiento de las relaciones sociales, sino además, en la regulación del comportamiento y en la motivación o conducta motivada de las personas (Izard, 1989).

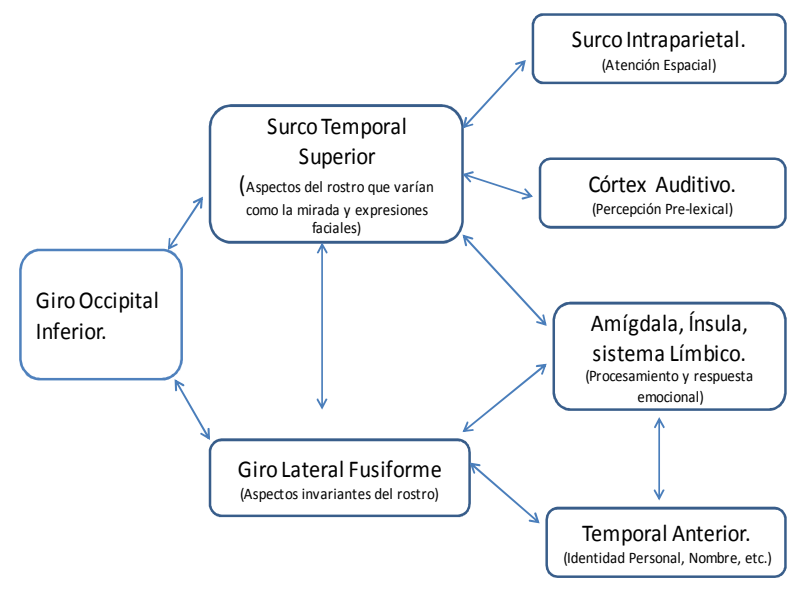

Figura 3. Modelo de la Distribución Neural para la Percepción de Rostros de Haxby

Tomando como referente lo anteriormente expuesto la presente investigación persigue describir las diferencias de rendimiento en tareas de memoria de rostros y el reconocimiento emocional en adultos mayores que no portan demencia, tanto institucionalizados como aquellos que conviven con sus familiares, residentes en la provincia de Villa Clara, Cuba.

\section{Método}

\section{Participantes}

En la investigación participaron un total de 100 adultos mayores de edades entre 65 y 70 años (Ver tabla 1), los cuales cumplían con criterios de selección tales como: no referir dificultades visuales severas, antecedentes de demencias, psicosis, traumatismos cráneo-encefálicos (TCE), accidentes cerebro-vasculares (ACV), o quejas de problemas de memoria y mostrar disposición para participar en la investigación. Un elemento importante es señalar que en todos los casos los ancianos habían vencido la educación media-superior. De estas personas, 50 convivían con sus familiares y el resto residen en asilos que se ubican en las ciudades de Santa Clara, Camajuaní y Caibarién, todas de la provincia de Villa Clara, Cuba.

Tabla 1. Comparación de la edad entre los grupos

\begin{tabular}{cccccc}
\hline & & $\mathrm{N}$ & Media & DE & Sig. \\
\hline \multirow{2}{*}{ Edad } & Institucionalizado & 50 & 68,5400 & 3,27 & \\
\cline { 2 - 7 } & No Institucionalizado & 50 & 68,6800 & 2,95 &, 350 \\
\hline
\end{tabular}

Para este último grupo se agregó como exigencia inclusoria superar los tres años de institucionalización, en función de que los adultos mayores que fueran explorados se encontraran adaptados a las características del internamiento pues, se ha demostrado, que el período que necesitan los adultos mayores para adaptarse a las particularidades del contexto de internamiento puede influir en el rendimiento cognitivo. 


\section{Materiales}

CMTF (Cambridge Memory Test for Faces) Versión en Español

El Test de Reconocimiento de Rostros de Cambridge (CFMT por sus siglas en inglés) es una prueba de amplia utilización utilizada para evaluar la capacidad para memorizar rostro Fue creado en el año 2004 y actualizado en el 2006. Sus autores son Brad Duchaine y Ken Nakayama.

La versión en español de la prueba fue realizada en la Universidad Central de Las Villas (Broche, 2011) y cuenta con la evaluación favorable del autor original de la técnica y de sus de colaboradores de habla hispana del Instituto de Neurociencia Cognitiva de la Universidad de Cambridge, que comenzaron a utilizar la herramienta para evaluar pacientes cuya lengua materna es el Español.

El CFMT es una prueba automatizada que está disponible para Windows y MAC OSX. Consta de tres grupos de tareas que evalúan la capacidad de reconocer rostros memorizados previamente, en tres niveles que aumentan paulatinamente su grado de complejidad (Ver Fig. 4).

La prueba cuenta con una fase inicial donde el participante se familiariza con las exigencias y demandas. Seguidamente se le presenta la primera de las tareas donde se familiariza con un rostro y debe reconocerlo dentro de un grupo de caras también familiares. En una segunda tarea se debe memorizar un grupo de caras que luego aparecen acompañadas de otras completamente nuevas. Por último se presentan rostros memorizados que la persona debe discriminar dentro de imágenes contaminadas por ruido visual.
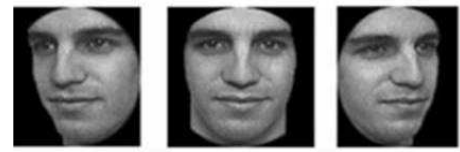

Ejemplos

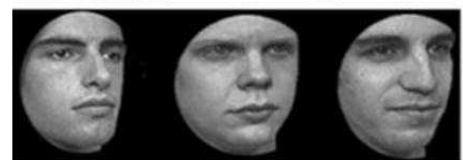

Tarea 1:Reconocimiento del rostro acompañado de rostros de familiaridad previa.

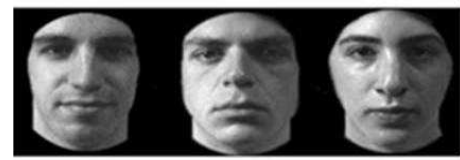

Tarea 2: Reconocimiento del rostro acompañado de rostros sin familiaridad.

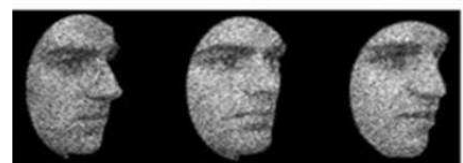

Tarea 3: Reconocimiento del rostro con ruido visual.

Figura 4. Muestra de las tareas de reconocimiento del Test de Reconocimiento de Rostros de Cambridge (CFMT)

Los valores son guardados automáticamente en un registro que contiene la respuesta ofrecida por el participante en cada ítem. Recoge si el resultado es correcto o incorrecto, el porciento de aciertos y los tiempos de reacción por cada ejecución, aunque para la presente investigación este último dato es irrelevante.

\section{International FACE Database}

La base de datos contiene fotografías de rostros de personas jóvenes, adultos y adultos mayores de ambos sexos, quienes representan diferentes emociones básicas (alegría, tristeza, miedo, asco, enojo). Este recurso, desarrollado por Michaela Riediger, cumple criterios de máximo control técnico, entiéndase por esto la utilización de vestuario, fondo, iluminación y resolución homogéneos. (Fig.5)

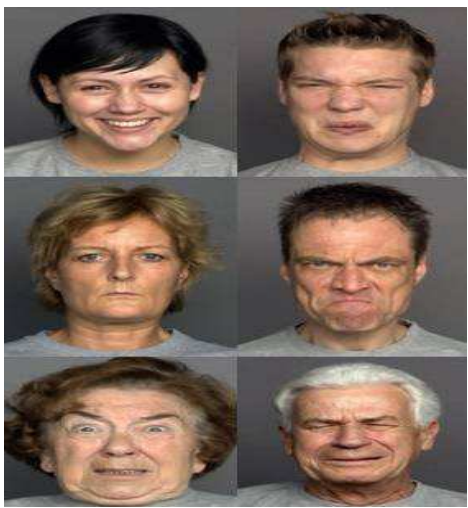

Figura 5. Muestra de las fotografías que contiene la International Face Database

Para el análisis y evaluación de las respuestas las fotos se acompañan de una etiqueta de identificación (ID) que contiene los datos relacionados con la emoción que expresa, el género y el grupo de edad al que pertenece.

\section{Procedimiento}

Una vez seleccionados los participantes con ajuste a los criterios anteriormente expuestos, se evaluó su capacidad de memorizar rostros y de reconocer emociones a través de los materiales descritos. Estos se aplicaron en el horario de la mañana, siendo necesarias un total de 36 sesiones de trabajo, desarrolladas desde Octubre de 2010 hasta Abril de 2011. Para la presentación de los exámenes automatizados se utilizó un ordenador portátil marca DELL, modelo Latitude C640, con una resolución de $1024 \times 768$ y sistema operativo Windows XP Service Pack 2. Estadísticamente los datos obtenidos se procesaron a partir de un análisis multivariado de varianza mediante procedimiento MANOVA, utilizando el paquete SPSS en su versión 18.0.

\section{Resultados}

\section{Análisis del CFMT}

Se realizó un análisis multivariado de varianza mediante procedimiento MANOVA para evaluar las diferencias existentes en la memoria de rostros entre los grupos estudiados considerando la misma como variable canónica integrada por tareas de reconocimiento simple, reconocimiento múltiple y reconocimiento con ruido.

Los resultados (Ver Tabla 2) revelaron que existen diferencias significativas entre los grupos en cuanto a la memoria de rostros. Los adultos mayores internos (AI) mostraron un mayor deterioro en esta variable, alcanzando valores que los diferencia del grupo de adultos que residen con sus familiares (NI) [Hotellings $=2,84, F \quad(3,97)=91,94$, $\mathrm{p}<.001]$. En este aspecto se obtuvo una sola función discriminante, donde el factor institucionalización (I) fue capaz de explicar el $73.98 \%$ de las puntuaciones obtenidas por 
los sujetos evaluados. El análisis de los centroides obtenidos entre los grupos reveló que los participantes AI tienden a mostrar valores superiores $(1,65)$ que los NI $(-1,68)$.

Una vez analizada la memoria de rostros como un todo deben analizarse los indicadores individuales de la prueba donde se observaron diferencias significativas para cada uno de ellos.

Tabla 2. Resultados CFMT

\begin{tabular}{llllllllllll}
\hline & \multicolumn{1}{c}{ AI } & \multicolumn{1}{c}{ NI } & \multicolumn{5}{c}{ Manova } \\
\hline & $\mathrm{M}$ & $\mathrm{D}$ & $\mathrm{N}$ & $\mathrm{M}$ & $\mathrm{D}$ & $\mathrm{N}$ & $\mathrm{gl}$ & $\mathrm{F}$ & $\mathrm{Si}$ & $\mathrm{y}^{2}$ & $\begin{array}{l}\text { Pote } \\
\text { ncia }\end{array}$ \\
& & $\mathrm{E}$ & & & $\mathrm{E}$ & & & & $\mathrm{g}$. & & \\
$\mathrm{R}$ & 7.9 & 2. & 5 & 15. & 2. & 5 & 1. & 270 & .0 & .7 & 1.00 \\
$\mathrm{~S}$ & 2 & 55 & 0 & 64 & 15 & 0 & 00 & .69 & 00 & 32 & 0 \\
\hline $\mathrm{R}$ & 10. & 4. & 5 & 18. & 5. & 5 & 1. & 68. & .0 & .4 & 1.00 \\
$\mathrm{M}$ & 44 & 36 & 0 & 43 & 29 & 0 & 00 & 30 & 00 & 08 & 0 \\
\hline $\mathrm{R}$ & 8.8 & 3. & 5 & 11. & 3. & 5 & 1. & 14. & .0 & .1 & 1.00 \\
$\mathrm{R}$ & 8 & 00 & 0 & 49 & 86 & 0 & 00 & 28 & 00 & 26 & 0 \\
\hline
\end{tabular}

Nota. RS (reconocimiento simple); RM (reconocimiento múltiple); $\mathbf{R R}$ (reconocimiento con ruido).

\section{Reconocimiento Simple}

El reconocimiento simple es la primera tarea que incluye el Test de Memoria de Rostros de Cambridge. Para la muestra estudiada se encontraron diferencias significativas entre los grupos $[\mathrm{F}(1,00)=270,69, \mathrm{p}<.001]$.

En el caso de los AI la media de las puntuaciones fue de 7,92 (SD = 2,55) mostrándose muy por debajo del grupo NI donde la media se comportó con valores de 15,64 (SD = 2,15). Los AI mostraron dificultades en 18 ensayos de la prueba, evidenciando de esta manera, mayor deterioro para memorizar rostros simples. El rendimiento de los NI fue muy superior comparado con sus iguales institucionalizados.

La potencia observada ${ }^{1}$ para la sección primera de la prueba (memoria simple de rostros) se comportó con un valor de 1.000. En este caso se demostró que la variable institucionalización influye de manera negativa y significativamente sobre la memoria de rostros $(\mathrm{p}<.001)$.

\section{Reconocimiento Múltiple}

En las tareas que comprende la segunda parte del CFMT (memoria múltiple) también se mostraron resultados que hablan de un mejor rendimiento de la memoria de rostros en el caso de los adultos mayores que conviven con sus familias. Comparativamente los AI mostraron un mayor deterioro que los NI, presentando dificultades para memorizar 23 ensayos. El análisis estadístico mostró que las diferencias entre los grupos es significativa $(\mathrm{p}<.001)$.

La media de las puntuaciones para los AI fue 10,44 $(S D=4,36)$ notándose, como en el caso del reconocimiento simple, por debajo de los valores alcanzados por el grupo NI, donde la media alcanzada fue de 18,43 $(S D=5,29)$. La potencia observada se comportó también con un valor de 1,000 .

La expresión de la memoria múltiple de rostros, junto con la memoria simple, indicó en sentido general un mayor deterioro en los adultos mayores que residen permanentemente en asilos, respecto a los que permanecen conviviendo con sus familiares $[F(1,00)=68,30, p<.001]$.

\footnotetext{
${ }^{1}$ Este valor se refiere a cuántas veces se obtendría el mismo resultado si se repitiera el estudio. En este caso se obtiene un valor de 1.000 indicando que de cada 100 veces que se replique el estudio en todos los caso se obtendría el mismo resultado.
}

\section{Reconocimiento con Ruido Visual}

El reconocimiento con ruido visual es la tercera y última de las tareas que contiene el CFMT. En ella deben memorizarse rostros que luego deberán discriminarse dentro de un conjunto que aparece contaminado visualmente. En esta parte de la prueba también fueron encontradas diferencias significativas entre los grupos estudiados $(p<.001)$. Los AI mostraron una media de las puntuaciones de 8,88 $(\mathrm{SD}=3,00)$ inferior a los adultos mayores que no se encuentran internados, donde la media se comportó de manera superior con un valor de $11,49(\mathrm{SD}=3,86)$.

Esta tercera fase complementa los hallazgos obtenidos en las etapas 1 y 2 del CMTF. Los resultados hablan a favor de una mayor conservación de la memoria de caras en los adultos que están rodeados por sus familiares, existiendo un deterioro significativo en la función de memoria de caras en el caso de los senescentes que se encuentran institucionalizados de manera permanente, y con un tiempo superior a los tres años, aun cuando no portan signos o síntomas demenciales.

La potencia observada para la última dimensión medida por el CFMT alcanzó un valor de 1.000. Como se constató en el reconocimiento simple y múltiple, también en el reconocimiento con ruido visual, la variable institucionalización influye en el deterioro de la memoria de rostros $[\mathrm{F}(1,00)=14,28, \mathrm{p}<.001]$.

Los resultados obtenidos en esta prueba se organizan en el gráfico que se expone a continuación (Ver Fig.3). En el mismo pueden compararse visualmente las diferencias expuestas durante el análisis del CFMT en sus tres dimensiones: reconocimiento simple, múltiple y con ruido visual.

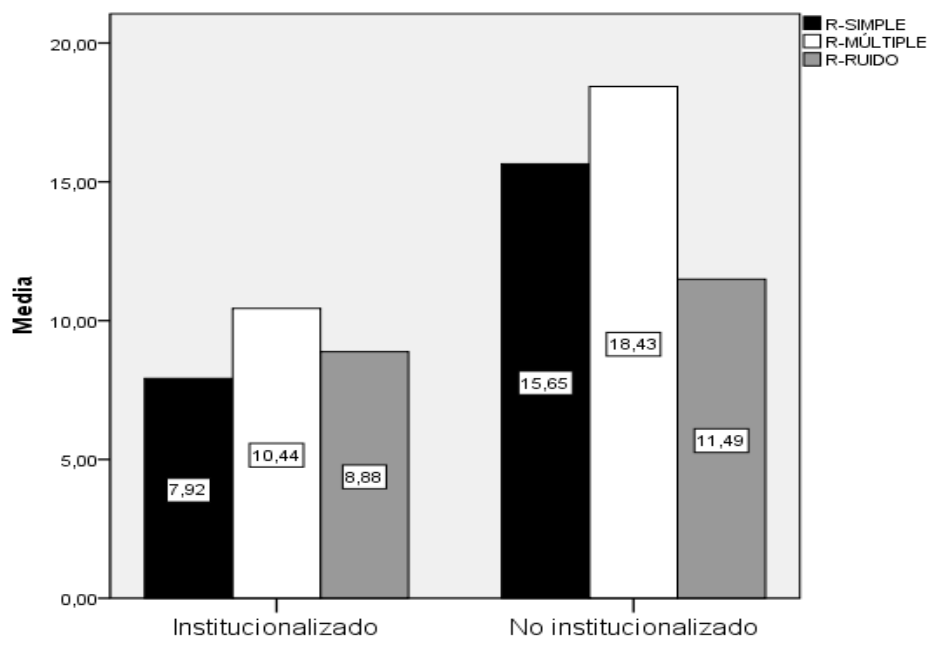

Figura 3. Resultados del CFMT

\section{Análisis de la Prueba de Reconocimiento Emocional}

El análisis de los datos obtenidos en la prueba de reconocimiento emocional se realizó igualmente con el empleo del análisis multivariado de varianza (MANOVA) para comparar las diferencias entre los grupos de AI y NI en cuanto al reconocimiento de emociones. De la misma manera que se procedió para el análisis de la memoria de rostros se decidió trabajar con una variable canónica. 
Luego de procesar los datos, los resultados revelaron que existen diferencias significativas entre los grupos estudiados en relación a la variable estudiada [Hotellings=2,00, F $(3,97)=64,73, \mathrm{p}<.001]$. Se comprobó que la capacidad para reconocer emociones está más preservada en los NI, mostrando los AI mayor deterioro en las tareas presentadas. Como resultado se obtuvo una sola función discriminante, donde el factor institucionalización fue capaz de explicar el $66,9 \%$ de la varianza. El análisis de los centroides aportó en este caso para los AI valores superiores $(1,38)$ en relación a los NI $(-1,41)$ (Ver Tabla 3$)$.

Tabla 3. Resultados Reconocimiento Emocional

\begin{tabular}{l|l|l|l|l|l|l|l|l|l|l|l}
\hline & \multicolumn{3}{|c|}{$\mathrm{AI}$} & \multicolumn{5}{c|}{$\mathrm{NI}$} & \multicolumn{6}{c}{ Manova } \\
\hline & $\mathrm{M}$ & $\mathrm{D}$ & $\mathrm{N}$ & $\mathrm{M}$ & $\begin{array}{l}\mathrm{D} \\
\mathrm{E}\end{array}$ & $\mathrm{N}$ & $\mathrm{gl}$ & $\mathrm{F}$ & $\begin{array}{l}\text { Si } \\
\mathrm{g} .\end{array}$ & $\mathrm{j}^{2}$ & $\begin{array}{l}\text { Pote } \\
\text { ncia }\end{array}$ \\
\hline $\mathrm{R}$ & 23. & 2. & 5 & 29. & 2. & 5 & 1. & 185 & .0 & .6 & 1.00 \\
$\mathrm{E}$ & 00 & 77 & 0 & 68 & 13 & 0 & 00 & .31 & 00 & 52 & 0 \\
\hline $\mathrm{R}$ & 35. & 3. & 5 & 35. & 1. & 5 & 1. & .58 & .4 & .0 & .117 \\
$\mathrm{Ed}$ & 42 & 43 & 0 & 01 & 50 & 0 & 00 & & 48 & 06 & \\
\hline $\mathrm{R}$ & 35. & .8 & 5 & 35. & .2 & 5 & 1. & 2.2 & .1 & .0 & .322 \\
$\mathrm{G}$ & 76 & 2 & 0 & 94 & 3 & 0 & 00 & 8 & 34 & 23 & \\
\hline
\end{tabular}

Nota. RE (reconocimiento emocional); REd (reconocimiento edad); RG (reconocimiento género)

Independientemente de que para la función discriminante creada, la institucionalización sea un factor que explique las diferencias entre los grupos los análisis univariados revelaron que las mayores diferencias se encuentran en el reconocimiento de emociones.

No obstante en secciones siguientes se analizan de forma individual y detallada las tres dimensiones que mide la prueba: reconocimiento emocional, reconocimiento del género y reconocimiento de la edad.

\section{Reconocimiento Emocional}

Los adultos mayores en esta prueba debían reconocer la emoción que presentaban 36 fotografías donde aparecen representados mujeres y hombres, en tres grupos etáreos fundamentales: jóvenes, adultos y adultos mayores. La media de las puntuaciones del reconocimiento emocional en los AI fue de 23,00 ( $\mathrm{SD}=2,77)$ siendo inferior que en el caso de los NI donde la media se comportó con un valor de 29,68 (SD = 2,1). Las diferencias, que muestra el análisis de esta variable, se comportan de manera significativa $(\mathrm{p}<.001)$ cuando se analiza el grupo de AI respecto a los NI [F $(1,00)=185,31$, $\mathrm{p}<.001]$. Este resultado demuestra que la institucionalización es un factor que influye en el deterioro de la capacidad para reconocer de emociones.

Un análisis más detallado refleja que no existe deterioro en el reconocimiento de emociones positivas en los grupos estudiados. No ocurre igual con las emociones negativas (asco, miedo, ira, tristeza) y neutral, donde se muestran grandes dificultades que van desde la incapacidad para el reconocimiento de las emociones, o la tendencia a confundir unas con otras (miedo por ira, tristeza por asco, etc.). En el caso de este análisis la potencia observada tuvo un valor de 1,000 .

Como se aprecia las diferencias en su conjunto muestran una elevada significación $(\mathrm{p}<.05)$. Fueron los NI los que mostraron una mayor conservación de la capacidad para reconocer emociones, respecto a los AI en los que se comprobó un deterioro de esta función.

\section{Reconocimiento de la Edad}

La edad aproximada era también un elemento que debía ser decodificada a partir de las fotografías presentadas. Para ello se establecieron tres edades fundamentales que se distribuyeron en joven, adulto y adulto mayor. En el análisis de la variable canónica "Reconocimiento Emocional" no se muestra diferencias significativas $(p>.05)$ en lo que respecta al reconocimiento de la edad, sin embargo, un análisis individual por fotografías sí mostró diferencias significativas $(\mathrm{p}<.05)$.

De manera general se presentaron 36 fotografías entre jóvenes, adultos y adultos mayores. Los AI presentaron dificultades para reconocer la edad aproximada en 19 del total, lo cual representa el $52,7 \%$ de ellas. Las dificultades se agruparon de la siguiente manera: las fotografías 1, 3, 4, 7, 25, referida a jóvenes; las fotografías $2,11,23,32,40,41,61$, que representan adultos y las fotografías 18, 23, 33, 36, 49 y 79 que presentan adultos mayores.

En todos estos casos los cálculos estadísticos muestran un nivel de significación alto ( $\mathrm{p}$. <05) cuando se comparan las respuestas de los AI y de los NI, mostrándose una mejor capacidad para decodificar la edad en el caso de los gerontes que no están institucionalizados. En el caso de la tercera de las variables medidas por la prueba (reconocimiento del género) no mostraron diferencias entre los grupos estudiados.

\section{Reconocimiento de Género}

En la prueba de reconocimiento del sexo ambos grupos presentaron un rendimiento normal sin presentar errores, no encontrándose diferencias ( $\mathrm{p}>.05)$.

Para poseer una idea global de la información obtenida con la prueba de reconocimiento de emociones se presenta un gráfico (Ver Fig. 3) que recoge los resultados alcanzados con el instrumento, comparándose las muestras estudiadas (adultos mayores institucionalizados y no institucionalizados).

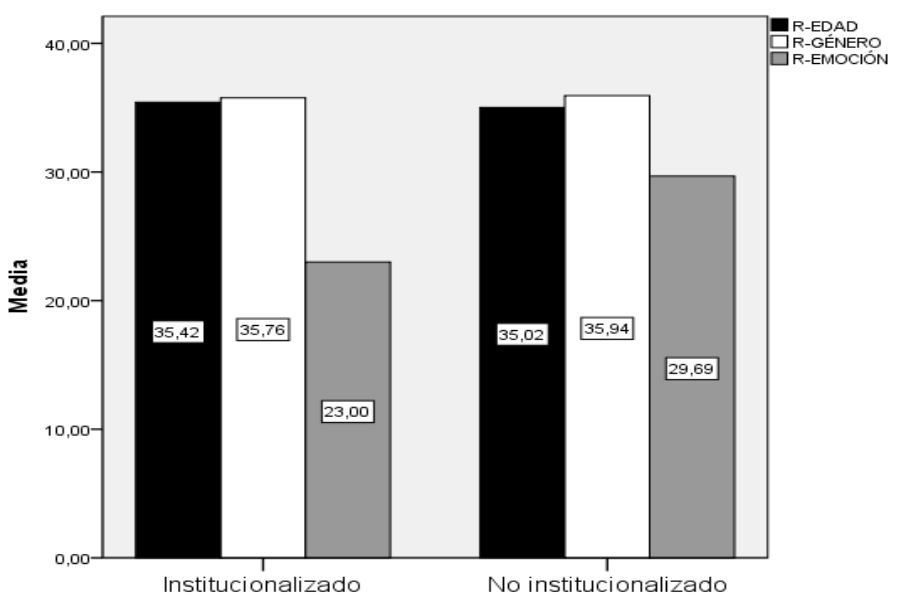

Figura 3. Resultados del Reconocimiento Emocional

\section{Discusión de los resultados}

El análisis de los datos obtenidos en las pruebas aplicadas permitió valorar, la coincidencia o diferenciación, de los resultados de la presente investigación con hallazgos reportados en trabajos anteriores relacionados con el tema 
(Esposito, et al., 2000; Garrido, et al., 2008; Bad-Jaim, et al., 2009; Boaz \& Galit, 2010).

Por ejemplo, en el caso específico de la memoria en ancianos institucionalizados, existe una investigación precedente realizada por Pérez, et al., (1995) (llevada a cabo en las Universidades de Granada y Universidad de Jaen) donde se exploró la memoria cotidiana y metamemoria en adultos mayores internos; en la misma se comparó la memoria de un grupo de ancianos que vivían en residencias respecto a un grupo de jóvenes.

Como resultado fundamental se obtuvo que los AI, en los resultados referidos a las tareas de memoria cotidiana, mostraron diferencias respecto a los jóvenes evidenciándose las mayores dificultades en nombres y caras; en el caso específico de los adultos mayores las puntuaciones fueron cuatro veces menor respecto al grupo comparativo.

Los elementos obtenidos en la presente investigación son compatibles con las conclusiones ofrecidas por Pérez (1995). Las puntuaciones obtenidas por nuestra muestra de AI se comportaron significativamente inferiores $(\mathrm{p}<.05)$ respecto a los NI. Debe destacarse que en este sentido no existe referencia de otro estudio que haya comparado la memoria de rostros en gerontes teniendo en cuenta la institucionalización, siendo este elemento uno de los mayores logros de la investigación.

Por otra parte resultó interesante observar cómo las diferencias entre grupos fueron haciéndose menores a medida que aumentaba la complejidad de la prueba. Estos resultados guardan relación con los estudios realizados en la Universidad Complutense de Madrid por el profesor Manzanero (2009), donde evaluó la memoria de rostros en adultos mayores sanos $y$ enfermos de Alzheimer, encontrando que las mayores diferencias se mostraban cuando reconocían rostros aislados, aumentando los errores cuando debían memorizar múltiples caras.

Al igual que en los resultados de Manzanero (2009) los adultos mayores participantes en la investigación mostraron mayores diferencias entre sí en la primera tarea (reconocimiento simple) que en las restantes (reconocimiento múltiple y con ruido).

Estos resultados muestran por primera vez el impacto directo de la institucionalización en la memoria de rostros. Si bien estudios anteriores relacionaron las categorías institucionalización, adultos mayores y memoria los datos obtenidos no ofrecieron una visión clara de su relación ni tampoco pudieron concluir que fuera exactamente el internamiento la causa del deterioro mnémico y no otra variable.

Como se muestra en los resultados del presente estudio, se debe reflexionar sobre la existencia de niveles de deterioro en el reconocimiento de rostros, lo que es compatible con la idea general del deterioro cognitivo leve, pudiéndose presentar indicios de dificultades gnósticomnémicas en poblaciones que no presentan funcionamiento deficitario. Un ejemplo de esto se tiene, cuando se analiza que el grupo de ancianos institucionalizados que fue objeto de estudio, no presentó puntuaciones en el Minimental que indicaran DCL o funcionamiento demencial, sin embargo presentaron deterioro en la memoria de rostros. En parte, esta dificultad también guarda relación con la naturaleza de las pruebas que se emplean para el diagnóstico del funcionamiento cognitivo general en la tercera edad, que habiltualmente no miden funciones específicas como la memoria de rostos o la capacidad de reconocimiento emocional.

Por otra parte, en las tareas de reconocimiento emocional los resultados se comportaron de acuerdo a una serie de investigaciones en este campo (Crook, \& Larrabee, 1992; De Haan, 2001; García, et al., 2008). Las mayores dificultades se presentaron en el reconocimiento de emociones negativas (ira, miedo, tristeza y asco), así como para los rostros neutrales; guardando relación con resultados obtenidos en otras investigaciones.

Por ejemplo García, et al., (2008), obtuvo resultados equivalentes en muestras similares al realizar un estudio, en la Universidad Autónoma de Barcelona, sobre el procesamiento emocional de las expresiones faciales en el envejecimiento normal y patológico. En sus resultados, demostraron que en el envejecimiento, tanto normal como patológico, hay un deterioro en el procesamiento emocional; especialmente de las emociones negativas como el miedo, la ira, la tristeza.

Debe señalarse que en los grupos estudiados se presentaron dificultades en el reconocimiento de la emoción marcando una diferencia significativa $(\mathrm{p}<.05)$ puesto que los institucionalizados obtuvieron puntuaciones muy inferiores a los no institucionalizados.

Este análisis permite aseverar que si bien en la vejez existe una dificultad general para el reconocimiento de emociones negativas, la institucionalización es un factor que agudiza este deterioro.

Otra información interesante estuvo relacionada con las dificultades que presentaron los AI en el reconocimiento de la edad aproximada de las personas que aparecían en las fotografías. Los adultos mayores debían precisar si la persona que mostraba la foto era un joven, un adulto, o un anciano (adulto mayor).

En este caso los miembros del grupo AI erraron en un número considerable de imágenes que contenían sujetos de todas las edades. Igualmente en este caso los NI mostraron mejor capacidad de reconocimiento respecto al grupo de AI. Junto a las dificultades para la memoria de rostros y el reconocimiento emocional, el reconocimiento inadecuado de la edad es un elemento que muestra deterioro en el caso de aquellos adultos mayores que se encuentran en instituciones de manera permanente.

Este hallazgo puede estar relacionado con variables de interacción social. En el caso de los adultos que conviven con sus familiares existe permanencia de las redes de apoyo social que han acompañado al adulto mayor durante toda su vida, de modo diferente, aquel que se inserta en un contexto de institucionalización debe reestructurar sus estructuras mnémicas asociadas a los rostros, lo cual puede verse afectado por el deterioro neurológico propio de la etapa evolutiva. Elemento que debe profundizarse en futuras investigaciones.

De manera general, resulta importante distinguir y estudiar como variables socioculturales y de interacción social influyen en la preservación de procesos cognitivos como la memoria de rostros y el reconocimiento de emociones. En este sentido la institucionalización de ancianos es un factor que influye significativamente en la preservación o deterioro de tales procesos. También importante el potencial cognitivo, relacionado con el nivel escolar y entrenamiento de funciones en los adultos mayores.

Como señalara la Bueno (1995) de la Universidad de Salamanca, los resultados de las investigaciones en esta área 
indican que la mecánica cognitiva presenta pérdidas consistentes con el envejecimiento que se pueden paliar, en parte, con el entrenamiento. Desde esta perspectiva, podríamos considerar la educación gerontológica como un tipo de intervención que hay que proporcionar ineludiblemente a las personas mayores de nuestro entorno con el objeto de promover sus potencialidades cognitivas y, por consiguiente, su calidad de vida.

En las próximas investigaciones que se realicen con adultos mayores institucionalizados debe tenerse en cuenta, además, el impacto emocional que provoca el deterioro de la memoria de rostros y el reconocimiento emocional.

Es importante recordar que lo emocional y lo cognoscitivo no puede segregarse, ambos guardan una estrecha interrelación bidireccional. En este sentido los estudios orientados a la capacidad de reconocimiento emocional y memoria de rostros pueden constituir el puente que entrelace el funcionamiento emocional y el rendimiento cognitivo, no solo en adultos mayores, también en otros tipos de poblaciones.

\section{Conclusiones}

En sentido general podemos concluir que los adultos mayores institucionalizados mostraron deterioro de la capacidad de memorizar rostros en los diferentes niveles del CMTF (reconocimiento simple, múltiple y con ruido), mostrando diferencias altamente significativas respecto al grupo de adultos mayores que conviven con sus familiares. Encontramos además, un mayor deterioro en la capacidad para reconocer emociones en los adultos mayores institucionalizados, observando diferencias altamente significativas respecto al grupo de personas que permanecen en su hogar. Por otra parte las emociones en las que se notaron mayores dificultades en los adultos mayores institucionalizados fueron el miedo, la ira, el asco, la tristeza y neutral; entre los grupos se establecieron diferencias significativas que indican una mejor conservación en los adultos que conviven con sus familiares. Finalmente en la tarea de reconocimiento de la edad el número mayor de errores se presentó en los adultos mayores institucionalizados, estableciéndose diferencias significativas entre los grupos en estudio. Comprobándose que los adultos mayores que conviven con sus familiares conservan mejor su potencial cognitivo. No se apreciaron diferencias significas entre los grupos estudiados en relación con el reconocimiento de expresiones positivas y atendiendo al género.

\section{Referencias}

Bad-Jaim, Y., Saidel, T., \& Yovel, G. (2009). The rol of skin color in face recognition. Perception, 38, 145-148.

Ballesteros, S. (1999). Memoria humana: investigación y teoría. Psicothema, 11 (4), 705-723.

Boaz, S., \& Galit, Y. (2010). Why is the N170 enhanced for inverted faces? An ERP competition experiment. NeuroImage.

Broche, Y. (2011). Traducción y validación del Cambridge Faces Memory Test. Manuscrito no publicado .

Bruce, V., \& Young, A. (1986). Understanding face recognition. British Journal of Psychology, 77, 305--327.

Bueno, B. (1995). Potencial cognitivo y envejecimiento. Aula: Revista de Pedagogía de la Universidad de Salamanca (7), 203-214.

Crook, T., \& Larrabee, G. (1992). Changes in facial recognition memory across the adult life span. Journal of Gerontology: Psychological Sciences, 47 (6), 138-141.
De Haan, E. H. (2001). Face perception and recognition. En B. Rapp, The handbook of cognitive neuropsychology (págs. 75-99). Filadelfia: Psychology Press.

Duchaine, B., \& Nakayama, M. (2006). Developmental Prosopagnosia: a window to content-specific processing. Current Opinion in Neurobiology, (16), 166-173.

Esposito, D., \& Weksler, M. (2000). Brain aging and memory: New findings help differentiate forgetfulness and dementia. Recuperado el 22 de Enero de 2011, de http://www.findarticles.com/cf_dls/m2578/6_55/63260233 /p1/article.jhtml?term=memory+++AND++identity+AND +aging

García, B., Fusari, A., \& Ellgring, H. (2008). Procesamiento emocional de las expresiones faciales en el envejecimiento normal y patológico. Neurología, 46, 315-326.

Garrido, L., Duchaine, B., \& Nakayama, K. (2008). Face detection in normal and prosopagnosic individuals. Journal of Neuropsychology, (2), 119-140.

Haxby, J. V., Hoffman, E. A., \& Gobbin, M. I. (2000). The distributed human neural system for face perception. Trends Cognitive Science, (4), 223-233.

Izard, C. (1989) Basic emotions, relations among emotions, and emotion-cognition relations. Psychological Review, 99(36) 561-565.

Lopera, F. (2000). Procesamiento de Caras: Bases Neurológicas, Trastornos y Evaluación. Revista de Neurología, 30(5).

Manzanero, A. L., Grandes, I. Y., \& Jódar, J. (2009). Edad y experiencia en el conocimiento y la descripción de personas. Boletín de Psicología, 95, 87-98.

Manzanero, A. L. (2010) Procesos cognitivos en el reconocimiento de caras. En Manzanero, A. L., Memoria de Testigos (pág. 131-146). Madrid: Ed. Pirámide.

Pérez, M., Peregrina, S., Justicia, J., \& Godoy, J. (1995). Memoria cotidiana y metamemoria en ancianos institucionalizados. Anales de Psicología , 11 (1), 47-62.

Portellano, J. A. (2005). Introducción a la Neuropsicología. Madrid: McGraw-Hill. 